\title{
Engineering Remotely Triggered Liposomes to Target Triple Negative Breast Cancer
}

\author{
Alexandra Sneider ${ }^{1}$, Rahul Jadia ${ }^{2}$, Brandon Piel ${ }^{1}$, Derek VanDyke ${ }^{1}$, Christopher Tsiros ${ }^{2}$, Prakash Rai ${ }^{1,2} \bowtie$ \\ 1. University of Massachusetts Lowell, Department of Chemical Engineering, Francis College of Engineering, 1 University Ave, Lowell, MA 01854, \\ USA. \\ 2. University of Massachusetts Lowell, Biomedical Engineering and Biotechnology Program, 1 University Ave, Lowell, MA 01854, USA. \\ $\triangle$ Corresponding author: prakash_rai@uml.edu, Phone: (978) 934-4971.
}

(C) Ivyspring International Publisher. This is an open access article distributed under the terms of the Creative Commons Attribution (CC BY-NC) license (https://creativecommons.org/licenses/by-nc/4.0/). See http://ivyspring.com/terms for full terms and conditions.

Received: 2016.08.30; Accepted: 2016.10.17; Published: 2017.01.01

\begin{abstract}
Triple Negative Breast Cancer (TNBC) continues to present a challenge in the clinic, as there is still no approved targeted therapy. TNBC is the worst sub-type of breast cancer in terms of prognosis and exhibits a deficiency in estrogen, progesterone, and human epidermal growth factor 2 (HER2) receptors. One possible option for the treatment of TNBC is chemotherapy. The issue with many chemotherapy drugs is that their effectiveness is diminished due to poor water solubility, and the method of administration directly or with a co-solvent intravenously can lead to an increase in toxicity. The issues of drug solubility can be avoided by using liposomes as a drug delivery carrier. Liposomes are engineered, biological nanoconstructs that possess the ability to encapsulate both hydrophobic and hydrophilic drugs and have been clinically approved to treat cancer. Specific targeting of cancer cell receptors through the use of ligands conjugated to the surface of drug-loaded liposomes could lessen damage to normal, healthy tissue. This study focuses on polyethylene glycol (PEG)-coated, folate conjugated, benzoporphyrin derivative (BPD)-loaded liposomes for treatment via photodynamic therapy (PDT). The folate receptor is over expressed on TNBC cells so these liposomes are targeted for greater uptake into cancer cells. PDT involves remotely irradiating light at $690 \mathrm{~nm}$ to trigger BPD, a hydrophobic photosensitive drug, to form reactive oxygen species that cause tumor cell death. BPD also displays a fluorescence signal when excited by light making it possible to image the fluorescence prior to PDT and for theranostics. In this study, free BPD, non-targeted and folate-targeted PEGylated BPD-loaded liposomes were introduced to a metastatic breast cancer cell line (MDA-MB-231) in vitro. The liposomes were reproducibly synthesized and characterized for size, polydispersity index (PDI), zeta potential, stability, and BPD release kinetics. Folate competition tests, fluorescence confocal imaging, and MTT assay were used to observe and quantify targeting effectiveness. The toxicity of BPD before and after PDT in monolayer and 3D in vitro cultures with TNBC cells was observed. This study may contribute to a novel nanoparticle-mediated approach to target TNBC using PDT.
\end{abstract}

Key words: Contrast agent, drug delivery, oncology, targeted therapy, verteporfin, nanotechnology.

\section{Introduction}

Triple negative breast cancer (TNBC) has the worst prognosis of breast cancers, accounting for $10-28 \%$ of all breast carcinomas [1, 2]. TNBC is characterized by the lack of expression of estrogen, progesterone, and human epidermal growth factor 2 receptors, making hormone derived therapies ineffective. Current treatment options include chemotherapy, radiotherapy, biologics, and surgery all of which have negative side effects [2-4]. There still remains no clinically approved, targeted therapy for this disease. Creation of a targeted, non-invasive, non-toxic therapy that could diagnose and treat TNBC would save many lives.

Nanotechnology, specifically the field of nano-theranostics, has shown great promise for developing an effective therapy. Liposomes, 
biological nanoconstructs made of lipids that form a spherical vesicle with hydrophobic bilayer and hydrophilic core, are already used in the clinic [5]. This nanoparticle is a desirable material for in vivo applications as the material is non-toxic, biodegradable, and can encapsulate both hydrophilic and hydrophobic small molecules. Liposomes may deliver the encapsulated therapy via one of two methods: enhanced permeability and retention (EPR) effect or receptor-mediated endocytosis. The EPR effect is more commonly referred to as a passive uptake mechanism, whereby the liposome and the contents within diffuse through and accumulate within the increased porosity of cancer's notoriously leaky vasculature to a greater extent than normal vasculature [3-9]. Receptor-mediated endocytosis relies upon a specific targeting agent that binds to a receptor on a cell's surface and is then taken up into the cell [10-11]. Success of both delivery methods relies upon circulation time in the body. Conjugating a stealth mechanism to the surface of the liposome, i.e. polyethylene glycol (PEG), can increase circulation time in vivo by prolonging macrophage uptake [3, 11-15]. Additionally, the use of a targeting agent would ideally improve the selectivity of the nanoconstruct; in this study folate was used as TNBC is one of many cancers that overexpresses the receptor [16-19].

Photo-triggered theranostics show great promise for non-invasively treating a disease. Photodynamic therapy (PDT) utilizes visible or near-infrared (NIR) light to remotely trigger a photosensitive agent, referred to as a photosensitizer (PS), to produce a reactive oxygen species (ROS) that can then induce cell death [20-27]. Verteporfin, also known as benzoporphyrin derivative monoacid, (BPD) is a desirable PS that has already been clinically approved for the treatment of macular degeneration; and is one of many PSs in use for the treatment of various diseases and cancers including dermatological, ophthalmic, brain, small cell lung, head and neck, gastroenterological, urological, and gynecological cancers [28]. BPD is a hydrophobic drug designed to target mitochondria that is non-cytotoxic unless irradiated with $690 \mathrm{~nm}$ light [23], making it an ideal therapeutic agent for selective targeting using a focused laser. BPD like most PSs can be used for optical fluorescence imaging when excited with the right wavelength of light and can therefore provide image-guided drug delivery, theranostics and PDT.

The engineered nanoconstruct in this study should target TNBC specifically in two ways: through the surface targeting of the folate over-expression in cancerous cells, and through the remote activation of the encapsulated BPD using PDT to trigger cell death.
The materials were specifically chosen with the aim of fast clinical approval, given that the individual components are already approved for the treatment of other diseases.

\section{Materials and Methods}

\section{Materials}

1,2-distearoyl-sn-glycero-3-phosphocholine (DSPC), cholesterol, 1,2-Distearoyl-sn-glycero-3-phosphoethanolamine-polyethylene glycol (DSPE-PEG, PEG MW =2000), DSPE-PEG 2000 folate were purchased from Avanti Polar Lipids Inc. (Alabaster, $\mathrm{AL}$ ), benzoporphyrin derivative (BPD) was obtained from Sigma Aldrich (St. Louis, MO), acetone was ordered from Acros Organics (Waltham, MA), chloroform was brought from Sigma Aldrich (St. Louis, MO). All chemicals obtained were analytical grade and were used without any further purification.

\section{Methods}

\section{Nanoparticle synthesis}

The desired chemicals were combined in a vial (DSPC, cholesterol, DSPE-PEG 2000 (non-targeted) or DSPE-PEG 2000-folate (targeted) 2:1:0.2, BPD $5 \%$ by weight); the organic solvents were initially evaporated using nitrogen gas; and then the vial was placed in a desiccator for 2 hours. Deionized water was preheated to $60^{\circ} \mathrm{C}$, and then the desired amount was added to the vial. The vial was closed and placed in a $60{ }^{\circ} \mathrm{C}$ water bath for 24 hours. A nitrogen gas powered extruder was used to transform the solution from multi-lamellar vesicles into small uni-lamellar vesicles, or liposomes, using a $0.1 \mu \mathrm{m}$ membrane. The solution underwent 11 cycles in the extruder. Post-extrusion the liposomal solution was placed in dialysis, MWCO $20 \mathrm{kDa}$, in a deionized water bath for 24 hours (stirring 60-100 rpm). The solution was then removed from the dialysis membranes, pushed through a $0.2 \mu \mathrm{m}$ filter, and characterized. The empty non-targeted and targeted liposomes were synthesized using the same procedure excluding the addition of BPD.

\section{Characterization}

Size, PDI, and zeta potential. The hydrodynamic diameter, polydispersity index (PDI), and zeta potential were measured using a dynamic light scattering (DLS) machine (Malvern Zetasizer Nano - ZS90). For DLS, $100 \mu \mathrm{L}$ of the sample was diluted with $900 \mu \mathrm{L}$ of deionized water and the results were measured in a plastic cuvette at $25^{\circ} \mathrm{C}$.

Drug encapsulation efficiency. Drug encapsulation efficiency of the liposomes was determined using the UV-Vis spectroscopy setting on 
the ThermoScientific NanoDrop 2000c, which measures the drug content by recording the absorbance of the sample. Determination of the amount of drug present was essential for the subsequent in vitro tests. For determining drug encapsulation efficiency, $100 \mu \mathrm{L}$ of the sample was mixed with $700 \mu \mathrm{L}$ of a $1 \%$ Triton $\mathrm{X}$ in PBS solution. The amount of drug present varied per batch and by nanoconstruct type.

Transmission electron microscopy. The samples were prepared for TEM by taking $10 \mu \mathrm{L}$ of the undiluted sample and pipetting it onto the surface of a 400 mesh, copper Formvar film grid. The sample was allowed to sit for 5 minutes before excess sample was removed using a kimwipe. The sample was then left to sit for 30 minutes. After 30 minutes, $10 \mu \mathrm{L}$ of a 2 $\%$ phosphotungstic acid (PTA) solution in DI water was applied to the surface serving as a negative stain. The grid was allowed to sit for an additional 5 minutes and then excess was removed using a kimwipe. The sample was then allowed to sit for an additional 10 minutes before imaging using the Phillip's EM400T Electron Microscope.

Stability studies. Stability studies were conducted for various batches of the folate-targeted BPD loaded liposomes in 1X PBS (1:1) at both $4{ }^{\circ} \mathrm{C}$, clinical storage temperature, and $37^{\circ} \mathrm{C}$, human body temperature over a 29-day period. Size and zeta potential measurements were made at different time points to monitor colloidal stability.

Drug release kinetics. The samples were placed in dialysis membranes; MWCO $20 \mathrm{kDa}$ (Spectrum Labs, Rancho Dominguez, CA) in $1000 \mathrm{~mL}$ of 1X PBS at $37{ }^{\circ} \mathrm{C}$ under constant stirring. At certain time points, a small volume of sample was removed from the dialysis bag for measuring BPD concentration using spectrometry. The absorbance of each sample was measured using UV-Vis Spectroscopy at 24 hour intervals for 7 days. Cumulative drug release was calculated as a percentage of initial drug encapsulated in the nanoconstructs.

\section{In vitro studies}

\section{Monolayer cell culture.}

BPD uptake. Monolayer dishes of MDA-MB-231 cells were incubated at $37^{\circ} \mathrm{C}$ with $0.5 \mu \mathrm{M}$ of BPD, free or encapsulated within passive and active liposomes, for 90 minutes. After that time, the dishes were washed with 1X PBS prior to being imaged using the Cy5 filter on an EVOS fluorescent microscope to detect BPD uptake.

Folate competition. Two separate experiments were performed where two dishes were pre-incubated for 4 hours with $1.0 \mu \mathrm{M}$ folate, and then treated with $0.5 \mu \mathrm{M}$ BPD-loaded passive and active liposomes. A further monolayer test was conducted for the active BPD liposomes in which $1.0 \mathrm{mM}$ free folate was pre-incubated and simultaneously incubated with $0.5 \mu \mathrm{M}$ BPD for 30 minutes. BPD fluorescence was imaged using an EVOS microscope. Monolayer MTT and live/dead imaging after PDT. MDA-MB-231 cell culture dishes were incubated at 37 ${ }^{\circ} \mathrm{C}$ with $0.5 \mu \mathrm{M}$ of BPD, free or encapsulated within passive and active liposomes, for 90 minutes. Following the incubation time, the cells were washed with 1X PBS to remove excess drug. The cells were irradiated with laser light at $690 \mathrm{~nm}$ at a fixed fluence of $2.5 \mathrm{~J} / \mathrm{cm}^{2}$ using a laser source (Intense, model \#7404, North Brunswick, NJ). 24 hours following PDT, the cells were qualitatively analyzed using standard live/dead, calcein AM/ ethidium homodimer (Molecular Probes, Life Technologies) stains. The cytotoxicity results were also analyzed using 3-(4,5-dimethylthiazol-2-yl)-2,5-diphenyltetrazolium bromide (MTT) (Molecular Probes, Life Technologies) dye to perform a standard calorimetric cell viability test. In brief, MTT was dissolved in 1X PBS to make a $5 \mathrm{mg} / \mathrm{mL}$ stock. $150 \mathrm{uL}$ of MTT stock was added to the culture dishes containing $1.5 \mathrm{~mL}$ of media and incubated for 3 hours. Following the incubation, dimethylsulfoxide (DMSO) was used to dissolved the formazan crystal and the results were obtained by reading absorbance using a plate reader (Molecular Devices SpectraMax M2e) at 570/690 nm.

$3 D$ cell culture. The Matrigel ${ }^{\circledR}$ matrix (Corning) was diluted 50\% with media (DMEM:Ham's F12 1:1, $10 \%$ FBS, $1 \%$ penicillin/streptomycin) on ice. The diluted $500 \mu \mathrm{L}$ matrigel was spread evenly onto the surface of a chilled $35 \mathrm{~mm}$ glass-bottomed dish evenly using a sterile, chilled cell spreader, and incubated for $1 \mathrm{hr}$ at $37^{\circ} \mathrm{C}$. 100,000 MDA-MB-231 cells/mL were added to chilled media (above) containing 10\% matrigel and $10 \mathrm{ng} / \mathrm{mL}$ epithelial growth factor (EGF). $2 \mathrm{~mL}$ of cell suspension was added to each dish and incubated at $37^{\circ} \mathrm{C}, 5 \% \mathrm{CO} 2$. Cell culture media was replaced with fresh media every 2 days for at least 10 days [29].

3D live/dead imaging, uptake, PDT. MDA-MB-231 cell based 3D acini were dosed with 10uM of BPD, in its free and encapsulated forms. After two hours of incubation time, the 3D acini were irradiated with $690 \mathrm{~nm}$ laser source at $10 \mathrm{~J} / \mathrm{cm}^{2}$ fluence, while power density was maintained at $100 \mathrm{~mW} / \mathrm{cm}^{2} .24$ hours post PDT, the acini were treated using standard live/dead, calcein AM/ ethidium homodimer (Molecular Probes, Life Technologies) to image the effect of PDT on 3D cultures using a fluorescence microscope. 


\section{Statistical Analysis}

Data are presented as the average mean \pm standard deviation. The significance of the difference between treatment groups was evaluated using unpaired Student's two-tailed t-test. $\mathrm{P}<0.05$ was considered statically significant.

\section{Results}

Four liposomal nanoconstructs were engineered for the study: non-targeted, non-drug-loaded liposomes (passive empty liposomes); targeted, non-drug loaded liposomes (active empty liposomes); non-targeted, drug loaded liposomes (passive BPD liposomes); and targeted, drug-loaded liposomes (active BPD liposomes). A schematic of the synthesis and the difference between active and passive BPD-loaded liposomes can be seen in Figure 1. This study utilizes liposomes made of DSPC, cholesterol, and DSPE-PEG 2000 in a 2:1:0.2 ratio by weight. The PEG aids in the prolonged circulation of the nanoparticle in vivo. In the case of the targeted nanoparticle, folate is conjugated to the end of the DSPE-PEG chain. BPD, at a ratio of 5 weight percent to the lipid quantity, is encapsulated in the hydrophobic lipid bilayer. These four liposomal constructs could be reproducibly synthesized in the lab using the solvent evaporation-hydration procedure followed by gas extrusion through a 0.1 micron polycarbonate filter.

\section{Size, PDI, and Zeta Potential}

The ideal hydrodynamic liposomal size is 100 $\mathrm{nm}$ with a polydispersity index (PDI) of $<0.100$ to confirm SUV formation [30-31]. The size is important for circulation within the vasculature, and the PDI is an indication of the homogeneity of the sample. The four liposome types proved to meet the requirements with the following averages (hydrodynamic diameter, PDI): passive empty $110.0 \mathrm{~nm}, 0.045$; active empty $114.3 \mathrm{~nm}, 0.044$; passive BPD $105.7 \mathrm{~nm}, 0.102$; and active BPD $113.8 \mathrm{~nm}, 0.076$.

Transmission Electron Microscopy (TEM) was used to verify DLS findings. TEM utilizes an electron beam to image the nanoparticles. TEM images of both the active and passive BPD liposomes show the desired spherical shape and size as shown in Figure 2(A-B).

The DLS instrument is also used to determine the zeta potential of the sample. The zeta potential is an indirect measure of the surface charge of the nanoparticle. This value is important for determining the circulation time and potential immune response in vivo. A zeta potential of $\leq-30 \mathrm{mV}$ is ideal for therapeutic agents [32]. Again, the four liposomal constructs proved to meet the requirements with the following averages: passive empty $-30.3 \mathrm{mV}$, active empty $-27.8 \mathrm{mV}$, passive BPD $-30.6 \mathrm{mV}$, and active BPD $-30.6 \mathrm{mV}$.

An overview of these results can be seen in Table 1. Results presented are averages over 13 independent repeats of the synthesis procedure.

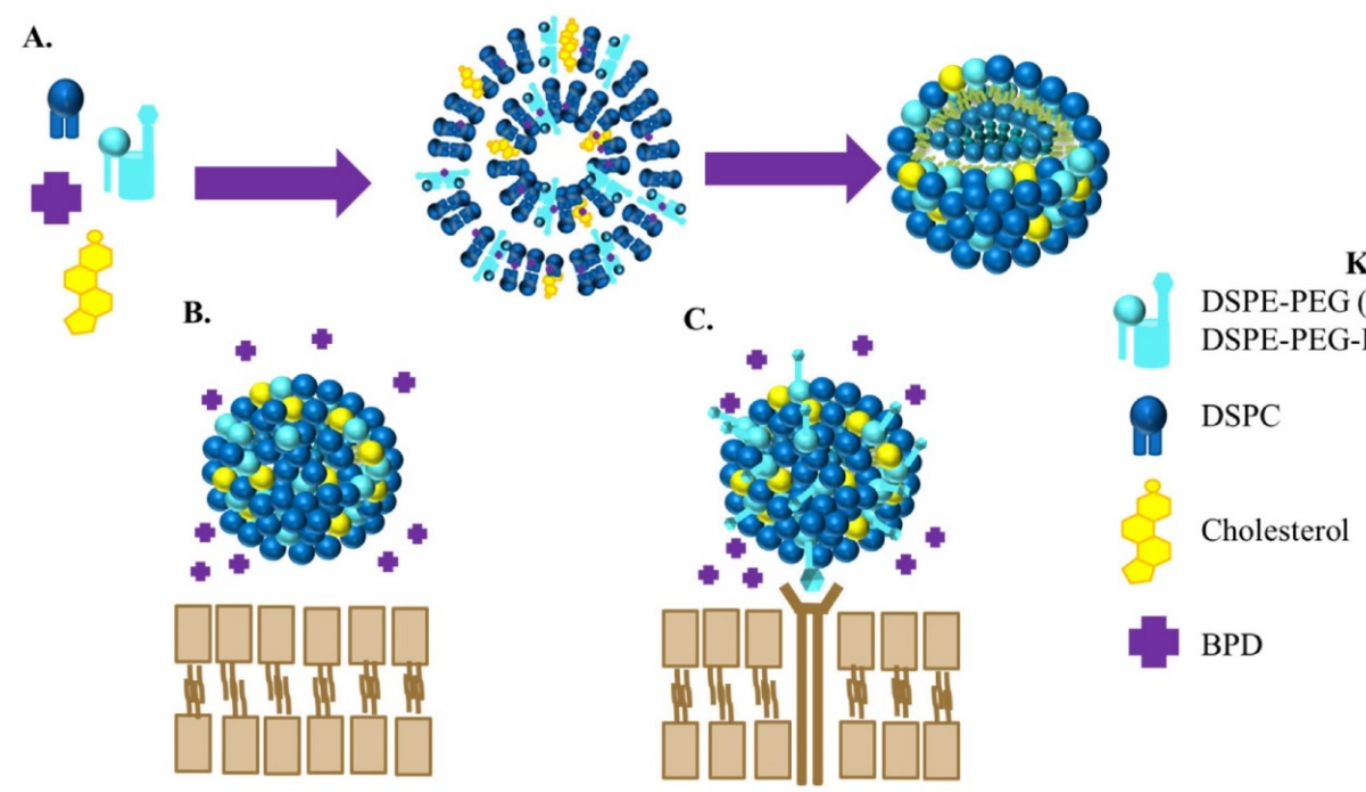

Figure 1. Cartoon of BPD loaded liposomes. (A) General procedure for the synthesis of BPD loaded liposomes. For passive targeted liposomes the lipid DSPE-PEG will be used in the synthesis whereas for active targeted liposomes the lipid used will be DSPE-PEG-Folate. (B) Non-targeted (passive) BPD- loaded liposome. (C) Folate-targeted (active) BPD liposome. 


\section{Stability Studies}

Stability studies were conducted for various batches of the active BPD liposomes in 1X PBS at both $4{ }^{\circ} \mathrm{C}$, clinical storage temperature; and $37^{\circ} \mathrm{C}$, human body temperature over a 29-day period. The biggest change in size and zeta potential occurred within the first 15 minutes of the sample mixing with PBS at both $4{ }^{\circ} \mathrm{C}$ and $37^{\circ} \mathrm{C}$. All experiments showed a relatively insignificant change in the hydrodynamic size of the nanoparticle, regardless of temperature. The experiments revealed a size change from roughly 107 $\mathrm{nm}$ to $101 \mathrm{~nm}$ during the study at both 4 and $37^{\circ} \mathrm{C}$ over the 29-day period (Figure 2C). The experiments also indicated a significant loss in the negative charge of the nanoparticle from roughly $-36 \mathrm{mV}$ to $-13 \mathrm{mV}$ at $4{ }^{\circ} \mathrm{C}$ and $-15 \mathrm{mV}$ at $37^{\circ} \mathrm{C}$ (Figure 2D).

\section{Drug Release Kinetics}

Drug release studies were conducted in 1X PBS at $37{ }^{\circ} \mathrm{C}$ over a 7 -day period to determine the effectiveness of the passive and active drug-loaded liposomes to retain the BPD. The BPD release studies showed that both the passive and the active BPD liposomes best fit the Korsmeyer-Peppas model with $\mathrm{R}^{2}$ value greater than 0.99 . The equation used for the Korsmeyer-Peppas model is as follows: \% release $=$ $\mathrm{k}_{\mathrm{KP}}{ }^{*} \mathrm{t}^{\mathrm{n}}$ where $\mathrm{k}_{\mathrm{KP}}$ and $\mathrm{n}$ are constants (for passive BPD-loaded liposomes $\mathrm{k}_{\mathrm{KP}}$ is 25.859 and $\mathrm{n}$ is 0.497 and for active BPD-loaded liposomes $\mathrm{k}_{\mathrm{KP}}$ is 44.301 and $n$ is $0.325)$. However, passive liposomes had a slower drug release rate showing $50 \%$ release by 3.5 days while active liposomes showed $50 \%$ release by 1.5 days. These results can be seen in Figure 2(E-F).

\section{In Vitro Studies}

Thus, having established the ability to consistently create all four of the liposomal constructs, the nanoparticles could then be used for a series of in vitro tests. This study utilized MDA-MB-231 cells (231s), a metastatic TNBC cell line.
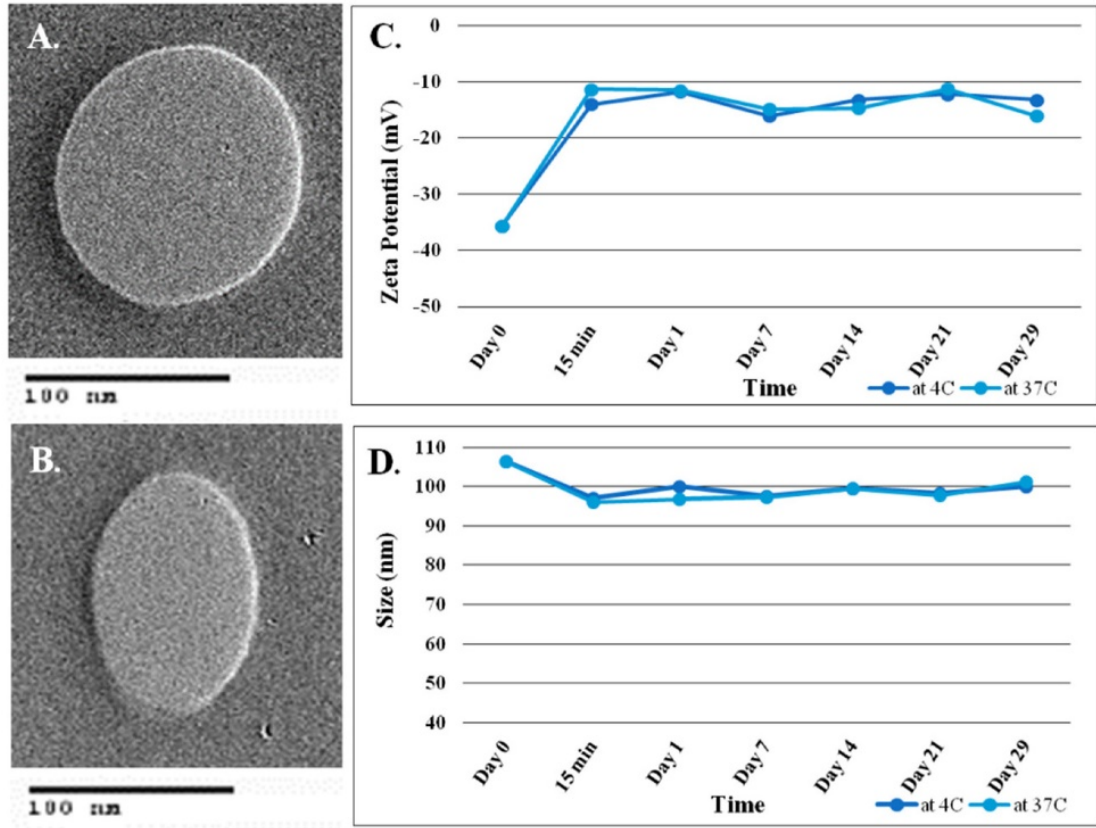
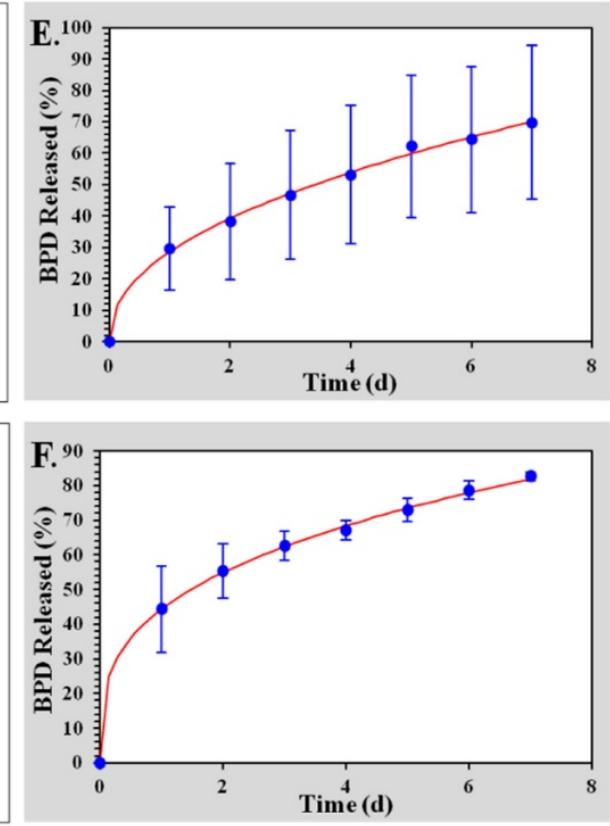

Figure 2. Liposome characterization. (A) TEM image of non-targeted BPD loaded liposome. (B) TEM image of folate targeted BPD loaded liposome. (C-D) Stability studies of folate targeted, BPD-loaded liposomes in IX PBS. (C) Zeta potential (mV) over time. (D) Hydrodynamic size (nm) over time. (E-F) Drug release studies using the Korsmeyer-Peppas model. (E) Passive BPD liposomes. (F) Active BPD liposomes.

Table 1. Physical Characterization of Non-targeted and Targeted Liposomes.

\begin{tabular}{llll}
\hline Liposomes & Size \pm SD $(\mathrm{nm})$ & PDI \pm SD & Zeta Potential \pm SD $(\mathrm{mV})$ \\
\hline Empty Non-Targeted $(\mathrm{n}=13)$ & $110.0 \pm 1.794$ & $0.045 \pm 0.020$ & $-30.3 \pm 1.706$ \\
Empty Folate-Targeted $(\mathrm{n}=13)$ & $114.3 \pm 1.419$ & $0.044 \pm 0.019$ & $-27.8 \pm 1.973$ \\
BPD-Loaded Non-Targeted $(\mathrm{n}=14)$ & $105.7 \pm 1.752$ & $0.102 \pm 0.015$ & $-30.6 \pm 2.723$ \\
BPD-Loaded Folate-Targeted $(\mathrm{n}=13)$ & $113.8 \pm 2.125$ & $0.076 \pm 0.020$ & $-30.6 \pm 1.604$ \\
\hline
\end{tabular}



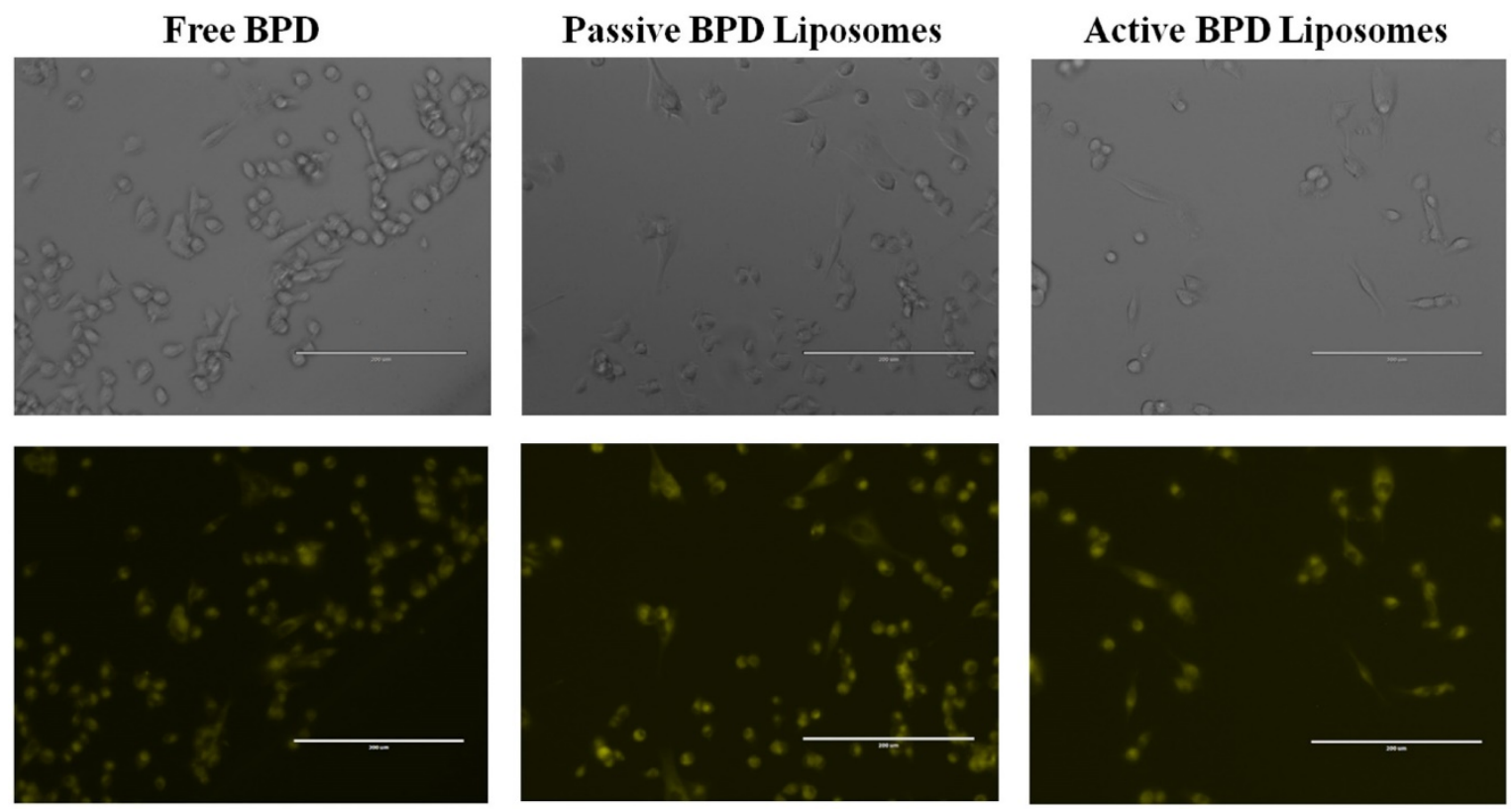

Figure 3. Monolayer BPD uptake. MDA-MB-231 cell line after 90 minutes incubation with $0.5 \mu M$ drug concentration. Images at $20 x$ using a fluorescent microscope. Fluorescent pseudo colors: Yellow - BPD. Scale bar: $200 \mu \mathrm{m}$.

\section{Monolayer cell culture}

BPD uptake. The first test was to see if the liposomes could deliver BPD to the 231s, and if the drug could be taken up by the cells. Incubation for 90 minutes proved similar fluorescence and successful uptake of BPD in 231s regardless of the drug delivery mechanism (Figure 3).

Folate competition studies. Additional folate competition tests were conducted in monolayer, in vitro to simulate in vivo conditions where there is competition between free folate and folate-conjugated liposomes for the receptors on the 231s. The aim of the experiment was to see if BPD could still be delivered successfully to the cells. Two dishes were pre-incubated with $1.0 \mu \mathrm{M}$ folate, and then $0.5 \mu \mathrm{M}$ BPD from passive and active BPD liposomes for 4 hours each. Fluorescent imaging showed that there was still BPD uptake, although at a lesser fluorescence, than when the cells are not pre-incubated with folate (Figure 4). A further monolayer test for the active BPD liposomes with a pre-incubation and simultaneous incubation of 1.0 $\mathrm{mM}$ free folate and $0.5 \mu \mathrm{M}$ BPD for 30 minutes still showed BPD uptake into 231s. These results indicate that even with folate competition, the engineered nanoconstructs may still successfully deliver BPD into the 231 s in vitro (Figure 5). For PDT purposes, it is not yet known whether the photosensitizer needs to be taken up into the cells or can be near the cells to effectively induce killing [33]. The reduction in BPD fluorescence from folate-targeted liposomes in these competition studies relative to the non-targeted liposomes confirmed the selective receptor-mediated update in the case of the targeted nanoconstructs.

Monolayer MTT and live/dead imaging, PDT. Having verified that the BPD does enter the 231s, the nanoconstructs (photosensitizer $0.5 \mu \mathrm{M}$ BPD) were incubated with the cells in monolayer, then irradiated with $690 \mathrm{~nm}$ light (fluence $2.5 \mathrm{~J} / \mathrm{cm}^{2}$, flux 200-220 $\left.\mathrm{mW} / \mathrm{cm}^{2}\right)$. The results were captured both qualitatively through imaging and quantitatively through MTT 24 hours after PDT. For the live/dead stain ethidium homodimer was used to show dead cells and calcein AM was used to show live cells under fluorescence imaging. Dark toxicity dishes of no treatment, free BPD, passive BPD liposomes, and active BPD liposomes showed that the cells were alive. This result was expected, as BPD is not supposed to be cytotoxic unless irradiated with light. PDT results, after irradiation show that the no treatment cells are alive, while the free BPD, passive BPD liposomes, and active BPD liposomes dishes are dead. Again, this result supports the notion that BPD in conjunction with PDT proves deadly to 231s. The photosensitizer alone or the light irradiation alone will not induce killing. The images with the stains simply show the difference between live and dead cells, but do not show differences in the cells due to the variation in drug delivery mechanism (Figure 6).

Morphological differences in the monolayer 231s 24 hours after PDT (fluence $5 \mathrm{~J} / \mathrm{cm}^{2}$, flux 200-220 $\mathrm{mW} / \mathrm{cm}^{2}, 0.5 \mu \mathrm{M}$ BPD) appear to be visible under 
fluorescent imaging based on the delivery mechanism. The dark toxicity dishes showed the cells to have a spindle shape, indicative of being alive and adhering to the dish regardless of the delivery mechanism. Post irradiation, the no treatment cells continue to have the spindle shape; while the morphologies of the cancer cells incubated with free BPD, passive BPD liposomes, and active BPD liposomes appeared to differ 24 hours after PDT. These differences could be attributed to the degree of cell death during the time frame; however, further testing is needed (Figure 7).

\section{Passive BPD Liposome Only}
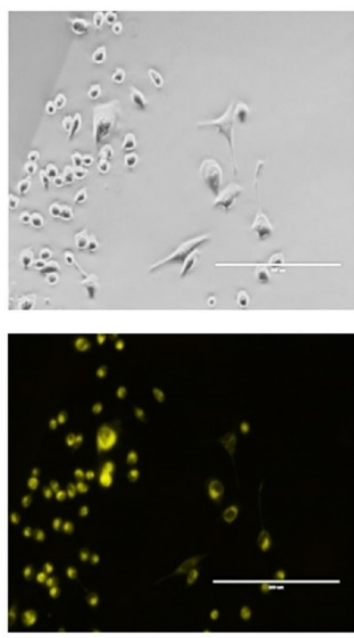

\section{Passive BPD} Liposome with Folate Competition
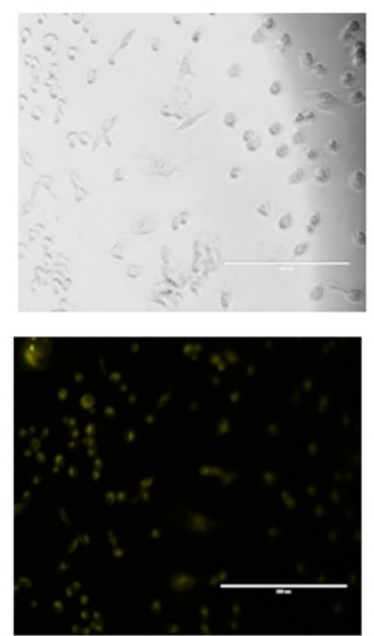

Active BPD Liposome Only
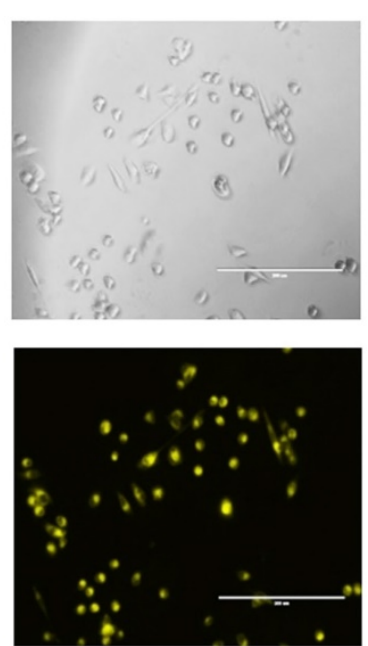

Active BPD Liposome with Folate Competition
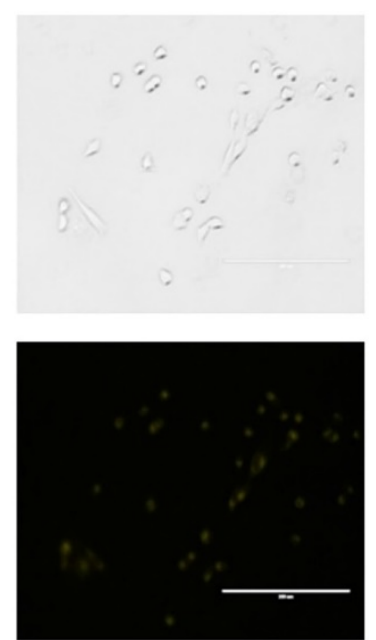

Figure 4. Folate competition of MDA-MB-231 cell line incubated for 4 hours. Incubated with $0.5 \mu$ M BPD and $1.0 \mu M$ Folate. No PDT. Fluorescent pseudo colors: Yellow - BPD. Scale bar: $200 \mu \mathrm{m}$.

Active BPD Liposome (ABL)
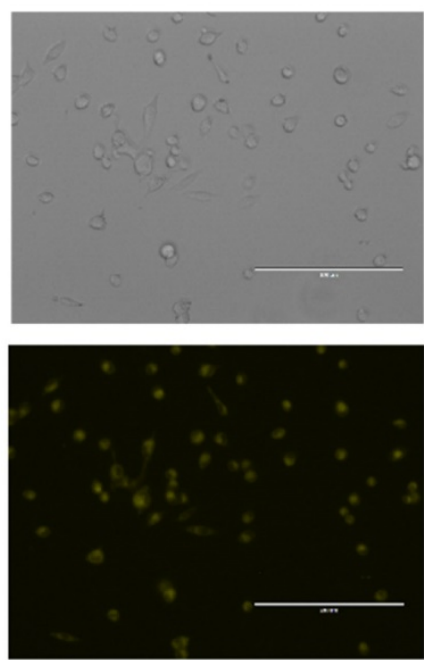

Folate and $A B L$
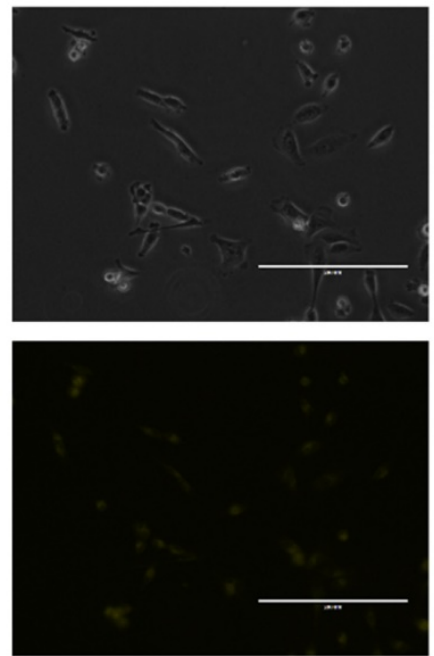

Folate Pre-Incubation
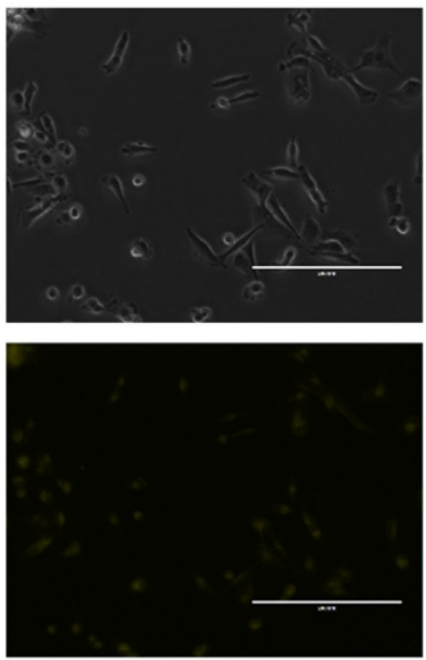

Figure 5. Folate competition of MDA-MB-231 cell line incubated for $\mathbf{3 0}$ minutes. Incubation using active BPD liposomes (0.5 $\mu$ M BPD), and 1.0 $\mathrm{mM}$ folate. Images at 20x a using a fluorescent microscope. Fluorescent pseudo colors: Yellow - BPD. Scale bar: $200 \mu \mathrm{m}$. 


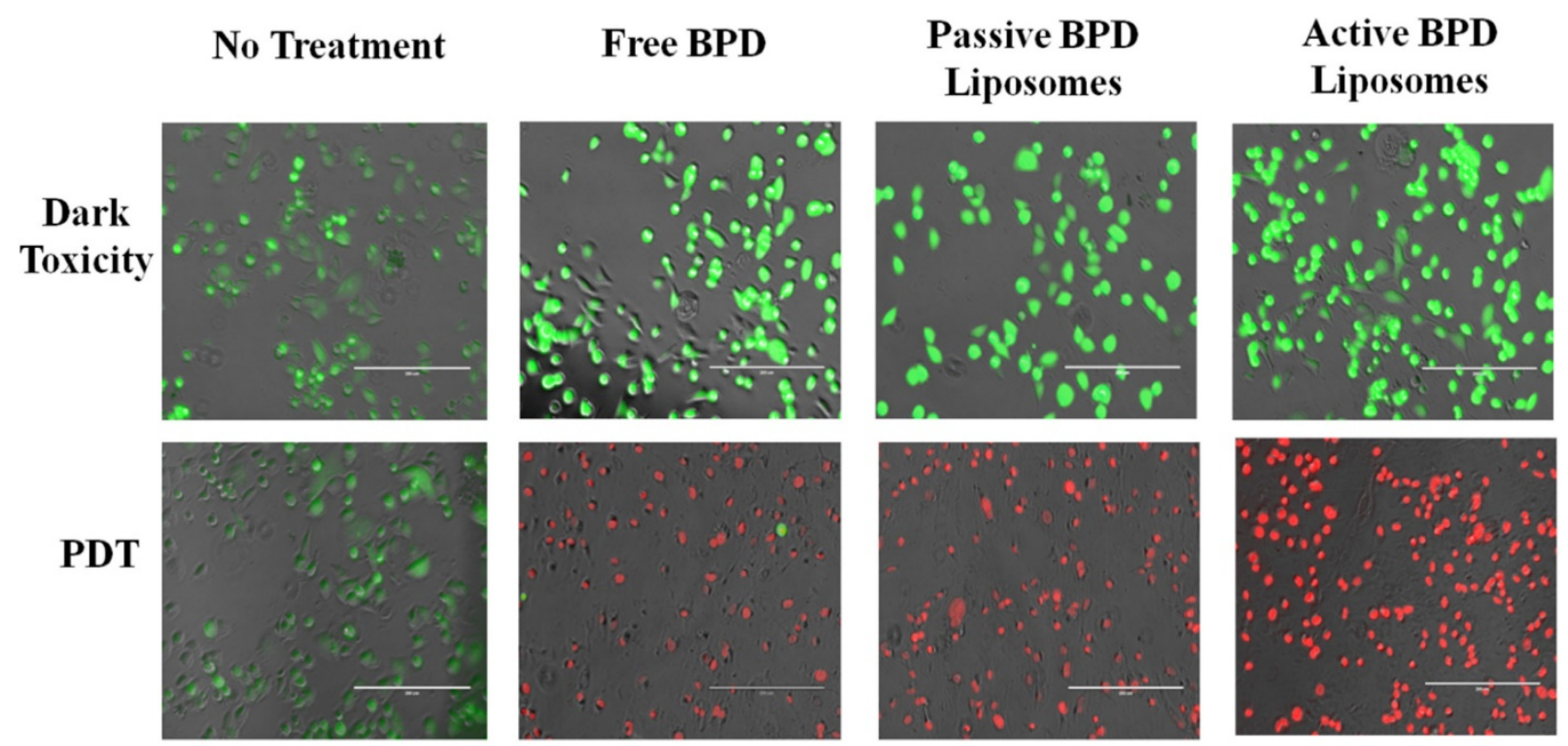

Figure 6. Live/dead cell fluorescence analysis of MDA-MB-231 cell line 24 hours after PDT. Fluence: $2.5 \mathrm{~J} / \mathrm{cm}^{2}$. Flux: $200-220 \mathrm{~mW} / \mathrm{cm}^{2}$. Concentration of photosensitizer: $0.5 \mu$ M. Fluorescent pseudo colors: Green - Live, Red - Dead. Scale bar: $200 \mu \mathrm{m}$.

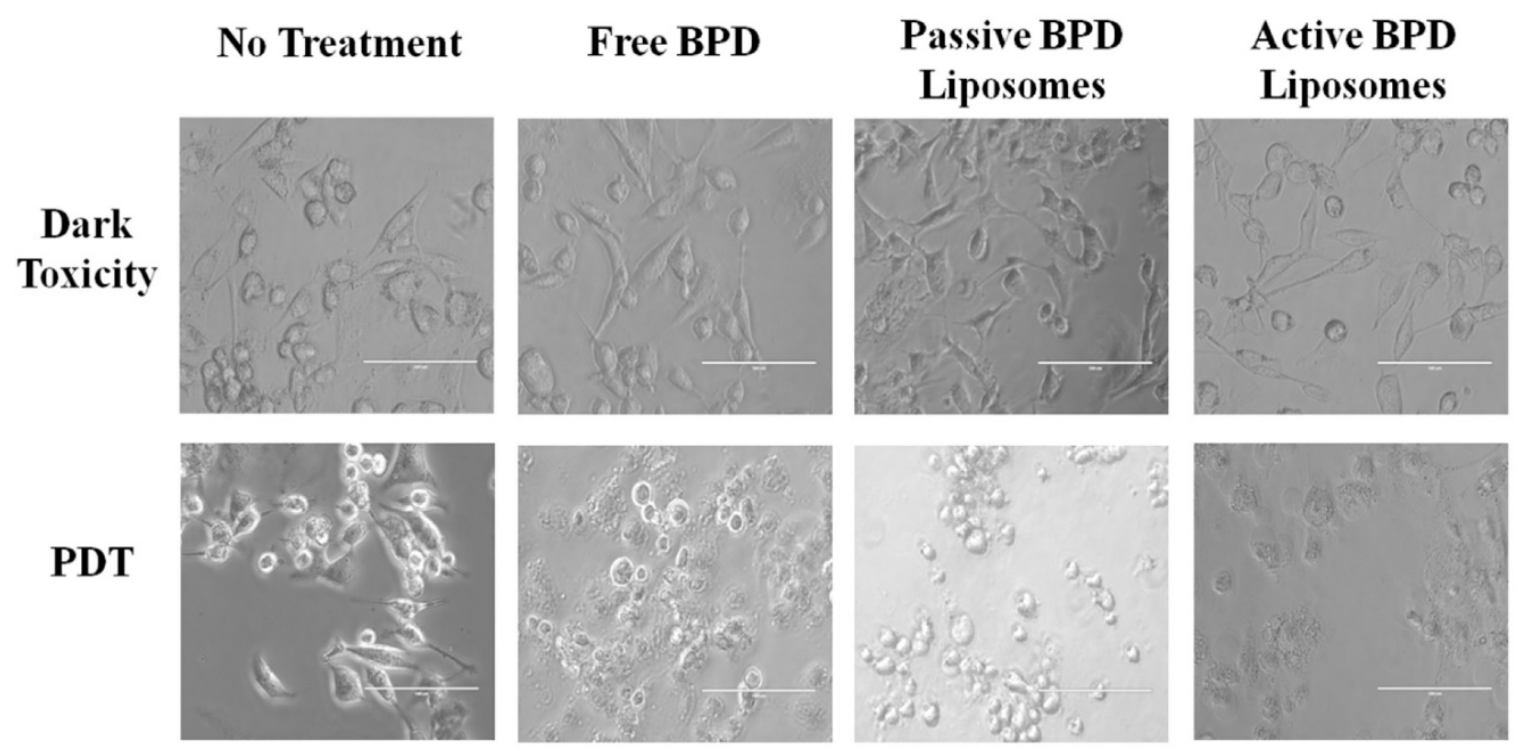

Figure 7. Cell morphology post PDT. MDA-MB-231 cell line 24 hours after photodynamic therapy. Fluence: $5 \mathrm{~J} / \mathrm{cm}^{2}$. Flux: $200-220 \mathrm{~mW} / \mathrm{cm}^{2}$. Concentration of BPD: $0.5 \mu \mathrm{M}$. Scale bar: $100 \mu \mathrm{m}$.

MTT is a quantitative, colorimetric assay to determine the percent cell viability by measuring the variations in absorbance from the reduction of tetrazolium dye to formazan by living cells. The no treatment, dark toxicity control is taken to be $100 \%$ cell viability, and $0 \%$ cell viability indicates complete cell death; these standards were then used to normalize the rest of the results. Four independent, monolayer killing experiments (fluence $2.5 \mathrm{~J} / \mathrm{cm}^{2}, 0.5$ $\mu \mathrm{M}$ BPD) were used for dark toxicity and PDT with no treatment, free BPD, passive BPD liposomes, and active BPD liposomes. Dark toxicity results show no treatment to have $100 \%$ cell viability, free BPD 103.3 $\%$, passive BPD liposomes $101.6 \%$, and active BPD liposomes $97.0 \%$. PDT results show no treatment (light only) $105.2 \%$, free BPD $33.8 \%$, passive BPD liposomes $33.0 \%$, and active BPD liposomes $33.3 \%$. These results are consistent with those observed using fluorescent microscopy; however, free $\mathrm{BPD}$, passive BPD liposomes, and active BPD liposomes seem to be producing the same killing results despite the varied delivery mechanisms. While it would have been 
desirable to see a marked separation in cell viability at this stage, there are shortcomings to monolayer, in vitro experiments (Figure 8) [34].

\section{D cell culture}

The next step was to repeat the killing experiments in a model that more closely resembles in vivo conditions, i.e. 3D cultures. 3D cultures of 231s were used on day 10, when the acini diameter leveled off between $100-120 \mu \mathrm{m}$ as seen in Figure 9 . Advanced fluorescent imaging was conducted for the dark toxicity and PDT (2 hr incubation, $10 \mu \mathrm{M}$ BPD, fluence $5 \mathrm{~J} / \mathrm{cm}^{2}$, flux $100 \mathrm{~mW} / \mathrm{cm}^{2}$ ) dishes to determine live/dead and BPD uptake. The images revealed similar trends to those observed in the monolayer experiments. The presence of some dead cells in the dark toxicity dishes is expected given the nature of the hypoxic core in tumors. While the combination of BPD and PDT continues to induce cell death, there are living cells in the acini regardless of the drug delivery method. Again, these results are to be expected (Figure 10) [35-37].

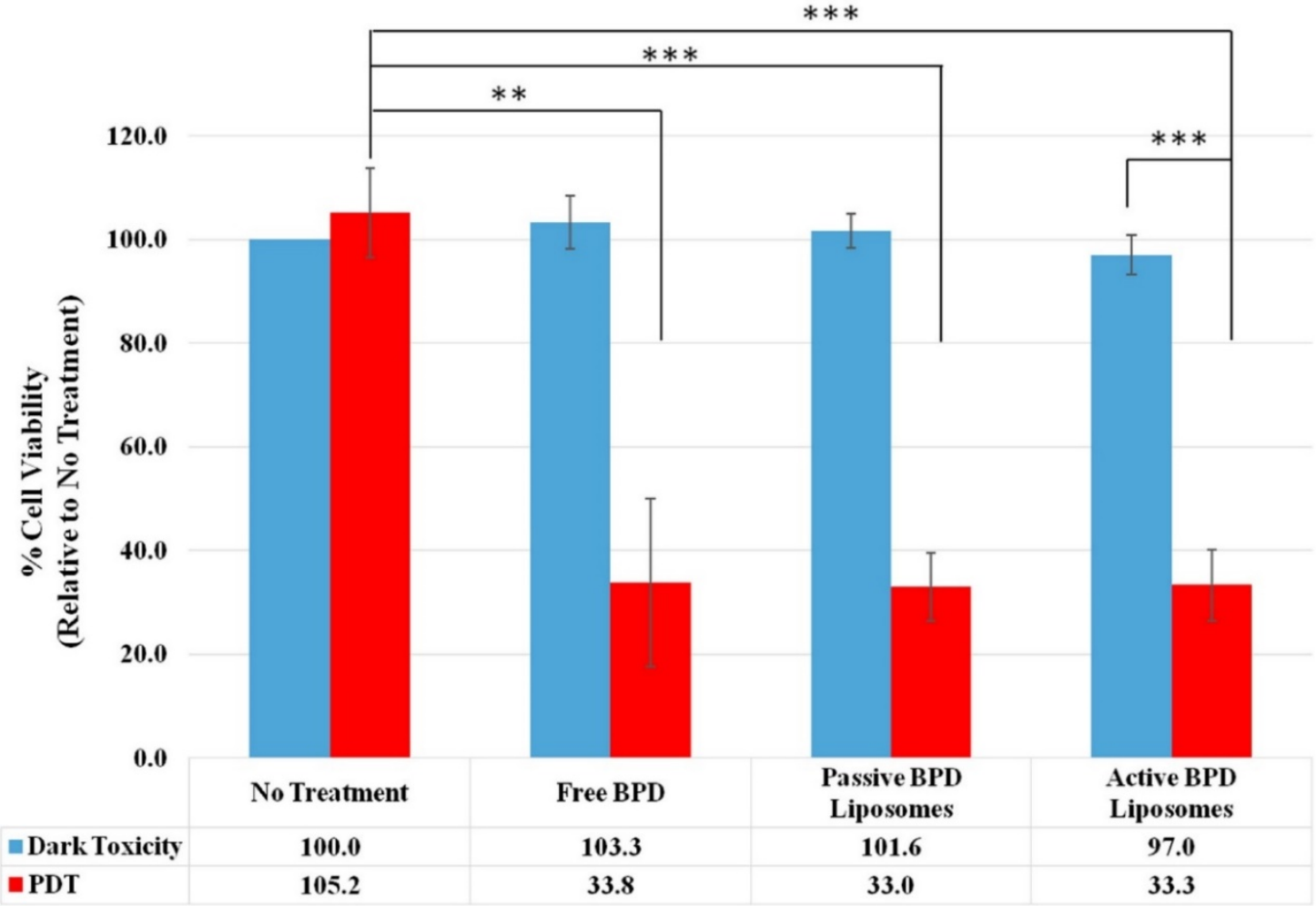

Figure 8. MTT assay 24 hours after PDT. Fluence $2.5 \mathrm{~J} / \mathrm{cm}^{2}, 0.5 \mu$ M BPD. $(\mathrm{n}=4, * * *=P<0.001, * *=P<0.01)$.
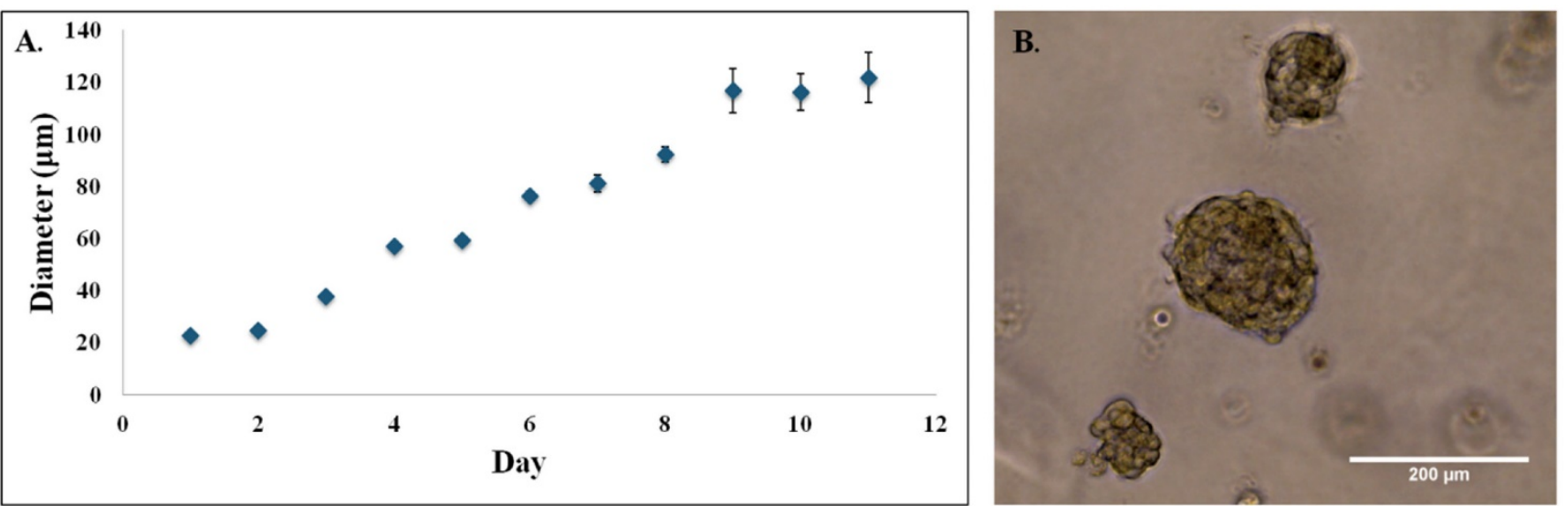

Figure 9. 3D MDA-MBA-231 acini. (A) 3D acini diameters estimated at 20x on light microscope for MDA-MB-231 cell line in 4-chamber dish. (B) 3D MDA-MB-231 acini on day 10. Image at 20x on light microscope. 


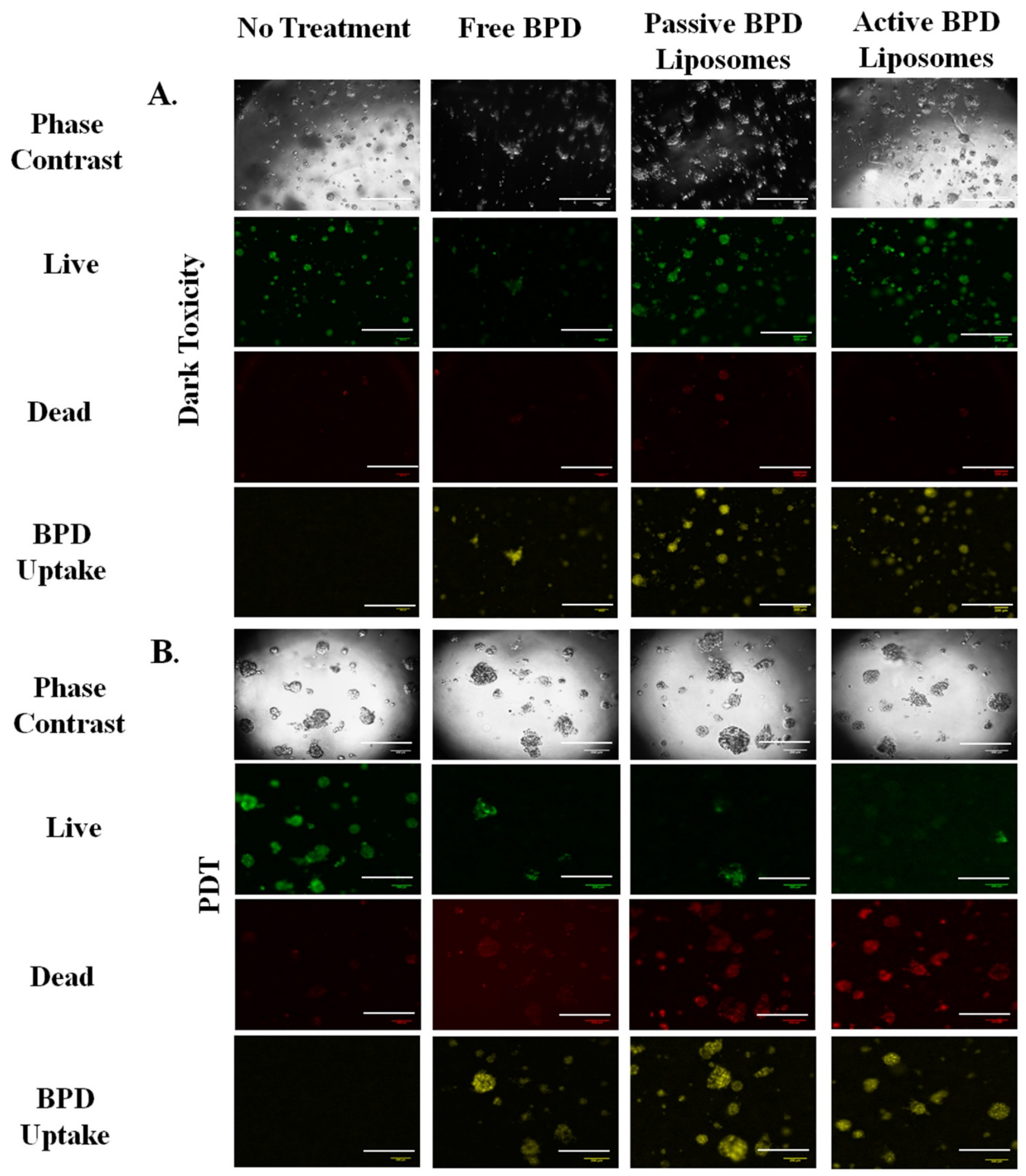

Figure 10. 3D Uptake Imaging. (A) Dark toxicity images 24 hours after incubation. Concentration of photosensitizer: $10 \mu \mathrm{M}$. Incubation time: $2 \mathrm{hr}$. Images all at $5 x$ using an advanced fluorescent microscope. (B) Uptake images 24 hours after photodynamic therapy. Fluence: $5 \mathrm{~J} / \mathrm{cm}^{2}$. Flux: $100 \mathrm{~mW} / \mathrm{cm}^{2}$. Concentration of photosensitizer: $10 \mu \mathrm{M}$. Incubation Time: $2 \mathrm{hr}$. Images all at $5 \mathrm{x}$ using an advanced fluorescent microscope. Fluorescent pseudo colors: Green - Live, Red - Dead, Yellow - BPD. Scale bar: $500 \mu \mathrm{m}$. 


\section{Discussion}

The choice of a liposomal nanoparticle and BPD were selected specifically with the end goal of clinical approval. The engineered liposomes target TNBC in two ways: with a folate ligand to actively select the folate receptor overexpression in TNBC, and with the photosensitive drug BPD to induce cancerous cell death. An additional advantage of the liposome is that it is designed not to be cytotoxic unless irradiated with $690 \mathrm{~nm}$ light; and may be remotely triggered, making it a non-invasive treatment method.

To verify the effectiveness of the folate-targeted, BPD-loaded liposomes, three other nanoconstructs were engineered as controls - non-targeted and folate-targeted empty liposomes and non-targeted, BPD-loaded liposomes. The synthesis process, replicated 13-14 times per construct, showed that the liposomes could be consistently reproduced with a desirable size of approximately $100 \mathrm{~nm}$, PDI less than 0.100 , and zeta potential $\leq-30 \mathrm{mV}$. TEM images of the drug loaded nanoparticles confirmed the desired spherical shape of the SUVs and that the non-hydrodynamic diameter was around the desired $100 \mathrm{~nm}$. These parameters are in line with already clinically approved nanoparticles [38].

Stability studies over a four-week period showed that a change in size and zeta potential of the nanoparticles occurs in PBS within the first 15 minutes at both clinical storage temperature $4{ }^{\circ} \mathrm{C}$ and human body temperature $37{ }^{\circ} \mathrm{C}$. The size change was relatively insignificant proving that the active $\mathrm{BPD}$ liposomes retained the desired dimensions for a period of a month. The change in zeta potential was significant and expected; despite this change, the surface charge did remain constant after the first 15 minutes. This change may affect the in vivo circulation time and therefore effectiveness of the nanoconstruct.

While stability over the four-week period was maintained, it is also important to consider the particles' ability to retain their therapeutic payload. Drug release kinetics studies showed that the active BPD liposomes release BPD faster than passive BPD liposomes. The increased interaction between water and folate molecule may increase the BPD release from the bilayer of targeted liposomes compared to non-targeted liposomes [39].

Having ensured that the liposomes were reproducible and would retain their physical characteristics, they were applied to monolayer MDA-MB-231 in vitro cell culture experiments. Introduction of the free BPD, passive and active BPD liposomes proved effective in delivering BPD to the 231s. Folate competition studies revealed that even with the pre-incubation or simultaneous incubation of free folate, the targeted and non-targeted BPD-loaded liposomes could still deliver BPD to the 231s. The significant reduction in BPD fluorescence in the 231s following incubation with the folate-targeted liposomes in these competition studies (relative to the non-targeted liposomes) confirmed the selective receptor-mediated update in the case of the targeted nanoconstructs.

Next, monolayer 231 dishes underwent PDT to induce killing. Fluorescent images confirm that only the combination of the photosensitizer BPD with the $690 \mathrm{~nm}$ irradiated light induced killing. The dark toxicity and light only dishes showed that the 231s were alive. While using the stain did not show any differences between the drug delivery methods, phase contrast images revealed morphological variations. These distinctions could be attributed to the degree of cell death induced during the 24-hour time period after PDT; further studies will need to be conducted to better understand the mechanism of cell death induced by the different constructs.

Colorimetric MTT assays semi-quantitatively confirmed the results seen in the fluorescent images. The free BPD, passive BPD liposomes, and active BPD liposomes actually all produced the same percentage of cell viability. While these results are not ideal, monolayer experiments with only 231 s are not the best model for determining the efficacy of the liposomes in vivo.

To improve upon the in vitro studies, PDT was conducted on a 3D cell culture model using 231s. The drug uptake, live and dead images appeared to be identical to those observed in the monolayer experiments. Optimization of the drug and PDT dose could improve these outcomes. Furthermore, the quantification of the fluorescent differences could provide insight on the comparative effectiveness of each method. These results confirm that the folate-targeted liposomes synthesized in this study could be effective in vivo and image-guided PDT using these targeted nanoconstructs could help reduce tumor burden while reducing collateral damage to healthy tissues.

\section{Conclusion}

The aim of this study was to create folate-targeted, BPD-loaded liposomal nanoconstructs that can target and treat TNBC, with the goal of being clinically approved in the future. Both the drug and the nanomaterial were chosen expressly for their impact already in the clinic. The folate ligand is desirable for targeting TNBC, and could be effective for treating other folate overexpressing cancers. 
Characterization using dynamic light scattering, TEM, and stability experiments reveal that the engineering nanoparticles are reproducible and stable. The stability studies demonstrated that the nanoparticles could be stored in the clinic for at least a period of a month, and retain their structure at $37^{\circ} \mathrm{C}$. The active BPD liposomes showed faster BPD release than the passive BPD loaded liposomes during a 7-day study.

In vitro studies indicate that the liposomes can effectively deliver BPD to MDA-MB-231 cells and induce killing with PDT in both monolayer and 3D cell culture studies. Monolayer MTT assay results suggest that the percent viability of $231 \mathrm{~s}$ was independent of the drug delivery method; however, this is not the best model to indicate in vivo efficacy. Better models include 3D cultures, co-cultures of MCF-12A and MDA-MB-231 cells, and in vivo studies. Furthermore, optimization of the PDT and drug dose could improve these results. Following the successful completion of in vivo studies, the folate-targeted, BPD-loaded liposomes show promise for a targeted, remotely triggered, clinically approved treatment option for TNBC and other folate-overexpressing cancers.

\section{Acknowledgements}

The authors acknowledge funding from the National Cancer Institute, a part of the National Institutes of Health (Award \# R00CA153948).

\section{Conflict of Interest}

The authors have declared that no conflict of interest exists.

\section{References}

1. Podo F, et al. Triple Negative Breast Cancer: Present Challenges and New Perspectives. Mol Oncol. 2010; 4: 209-229.

2. Guarneri V, Dieci M, Conte PF. Relapsed Triple Negative Breast Cancer: Challenges and Treatment Strategies. Drugs. 2013; 73(12): 1257-1265.

3. Milane L, et al. Multi-modal Strategies for Overcoming Tumor Drug Resistance: Hypoxia, the Warburg Effect, Stem Cells, and Multifunctional Nanotechnology. J Control Release. 2011; 155: 237-247.

4. Kwon IK, et al. Analysis on the Current Status of Targeted Drug Delivery to Tumors. J Control Release. 2012; 164: 108-114.

5. Paszko E, et al. Nanodrug Applications in Photodynamic Therapy. Photodiagnosis Photodyn Ther. 2011; 8(1): 14-29.

6. Hollis CP, Weiss HL, Leggas $M$, et al. Biodistribution and Bioimaging Studies of Hybrid Paclitaxel Nanocrystals: Lessons Learned of the EPR Effect and Image-Guided Drug Delivery. J Control Release. 2013; 172: $12-21$.

7. Maeda H, Wu J, Sawa T, et al. Tumor Vascular Permeability and the EPR Effect in Macromolecular Therapeutics: A Review. J Control Release. 2000; 65(1-2): 271-284

8. Torchilin VP. Targeted Pharmaceutical Nano Carriers for Cancer Therapy an imaging. AAPS J. 2007; 9(2): E128-E147.

9. Torchilin VP. Tumor Delivery of Macromolecular Drugs Based on the EPR Effect. Adv Drug Deliv Rev. 2011; 63(3): 131-135.

10. Bhatti M, Yahioglu G, Milgrom LR, et al. Targeted Photodynamic Therapy with Multiply-Loaded Recombinant Antibody Fragments. Int J Cancer. 2008; 122: 1155-1163.

11. Xie S, Tao Y, Pan Y, et al. Biodegradable Nanoparticles for Intracellular Delivery of Antimicrobial Agents. J Control Release. 2014; 187: 101-117.
12. Zhang L, et al. Nanoparticles in Medicine: Therapeutic Applications and Developments. Clin Pharmacol Ther. 2008; 83: 761-769.

13. Shen M, Huang Y, Han L, et al. Multifunctional Drug Delivery System for Targeting Tumor and its Acific Microenvironment. J Control Release. 2012; 161: 884-892.

14. Fontana G, Licciardi M, Mansueto $S$, et al. Amoxicillin-Loaded Poluethylcyanoacrylate Nanoparticles: Influence of PEG Coating on Particle Size, Drug Release Rate and Phagocytic Uptake. Biomaterials. 2001; 22: 2857-2865.

15. Dhule SS, Penfornis P, He J, et al. The Combined Effect of Encapsulating Curcumin and C6 Ceramide in Liposomal Nanoparticles Against Osteosarcoma. Mol Pharm. 2014; 11: 417-427.

16. Tacha D, Bremer R. Folate Receptor alpha is Frequently Expressed in Triple Negative Breast Cancers. Modern Pathol. 2013; 26(Supplement 2): $71 \mathrm{~A}$.

17. Necela BM, Crozier JA, Andorfer CA, et al. Folate Receptor-a (FOLR1) expression and function in triple negative tumors. PLOS ONE. 2015; 10(4): e0127133.

18. Zhang Z, Wang J, Tacha D, et al. Folate Receptor a Associated with Triple-Negative Breast Cancer and Poor Prognosis. Arch Pathol Lab Med. 2014; 138(7): 890-895.

19. O'Shannessy DJ, Somers EB, Maltzman J, et al. Folate Receptor Alpha (FRA) Expression in Breast Cancer: Identification of a New Molecular Subtype and Association with Triple Negative Disease. SpringerPlus. 2012; 1: 22.

20. Randles EG, Bergethon PR. A Photodependent Switch of Liposome Stability and Permeability. Langmuir. 2013; 29(5): 1490-1497.

21. Allison RR, Moghissi K. Oncologic Photodynamic Therapy: Clinical Strategies that Modulate Mechanisms of Action. Photodiagnosis Photodyn Ther. 2013; 10(4): 331-341.

22. Akens MK, et al. In vitro and in vivo Effects in Photodynamic Therapy on Metastatic Breast Cancer Cells Pre-Treated with Zoledronic Acid. Photodiagnosis Photodyn Ther. 2014; In Press.

23. O'Connor AE, Gallagher WM, Byrne AT. Porphyrin and Nonporphyrin Photosensitizers in Oncology: Preclinical and Clinical Advances in Photodynamic Therapy. Photochem Photobiol. 2009; 85: 1053-1074.

24. Josefsen LB, Boyle RW. Photodynamic Therapy: Novel Third-Generation Photosensitizers one step closer? Br J Pharmacol. 2008; 154: 1-3.

25. Huntosova V, Alvarez L, Bryndzova L, et al. Interaction Dynamics of Hypericin with Low-Density Lipoproteins and U87-MG Cells. Int J Pharm. 2010; 389: 32-40.

26. Leonard KA, Nelen MI, Simard TP, et al. Synthesis and Evaluation of Chalcogenopyrylium Dyes as Potential Sensitizers for the Photodynamic Therapy of Cancer. J Med Chem. 1999; 42: 3953-3964.

27. Bergstrom LC, Vucenik I, Hagen IK, et al. In-vitro Photocytotoxicity of Lysosomotropic Immunoliposomes Containing Pheophorbide a with Human Bladder Carcinoma Cells. J Photochem Photobiol B. 1994; 24: 17-23.

28. Huang Z. A Review of Progress in Clinical Photodynamic Therapy. Technol Cancer Res Treat. 2005; 4(3): 283-293.

29. Lee, G.Y. Three-dimensional culture models of normal and malignant breast epithelial cells. Nat Methods. 2007. 4(4): 359-65

30. Torchilin VP. Recent Advances with Liposomes as Pharmaceutical Carriers. Nat Rev Drug Discov. 2005; 4: 145-160.

31. Tang $L$, et al. Investigating the optimal size of anticancer nanomedicine. Proc Natl Acad Sci USA. 2014; 111(43): 15344-15349.

32. Honary S, Zahir F. Effect of Zeta Potential on the Properties of Nano-Drug Delivery System - A Review. Trop J Pharm Res. 2013; 12(2): 265-273.

33. Rai, P, et al. Development and applications of photo-triggered theranostic agents. Adv Drug Deliv Rev. 2010; 62(11): 397-9.

34. Edmondson R, Broglie JJ, Adcock AF, Yang L. Three-dimensional cell culture systems and their applications in drug discovery and cell-based biosensors. Assay Drug Dev Technol. 2014; 12(4): 207-218.

35. Wilson BC. Photodynamic therapy for cancer: principles. Can J Gastroenterol Hepatol. 2002; 16(6): 393-396.

36. Vrouenraets MB, Visser GW, Snow GB, van Dongen GA. Basic principles, applications in oncology and improved selectivity of photodynamic therapy. Anticancer Res. 2003; 23(1B): 505-522.

37. Capella MA, Capella LS. A light in multidrug resistance: photodynamic treatment of multidrug-resistant tumors. J Biomed Sci. 2003; 10(4): 361-366.

38. Abraham SA, et al. The liposomal formulation of doxorubicin. Methods Enzymol. 2005; 391: 71-97.

39. Mandal S, et al. Design, development and characterization of multi-functionalized gold nanoparticles for biodetection and targeted boron delivery in BNCT applications. Appli Radiat Isot. 2011; 69: 1692-7. 


\section{Author Biography}

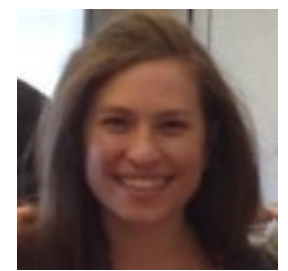

Alexandra Sneider obtained her bachelor's degree in Chemical Engineering from the University of Massachusetts Lowell in 2016, and has a previous BA in Anthropology and Archaeology from Harvard University in 2011. She will be starting her $\mathrm{PhD}$ in Chemical and Biomolecular Engineering at Johns Hopkins University in 2016. Alexandra was awarded a National Science Foundation Graduate Research Fellowship in 2016; American Institute of Chemists, Chemical engineering Student Award 2016; American Institute of Chemical Engineers, Outstanding Chemical Engineering Student Award 2015; American Chemical Society, Division of Colloids 250 th Conference Travel Award 2015; and Materials Research Society, First place Best Poster Winner Boston 2014.

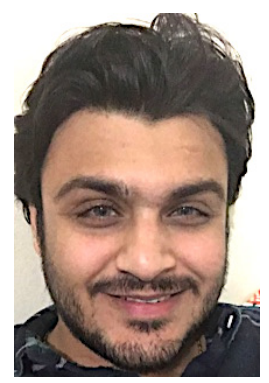

Rahul Jadia is a PhD student in Biomedical Engineering Program at University of Massachusetts Lowell, US. He earned his Master's in Plastics Engineering at University of Massachusetts Lowell in 2014 and received his Bachelor's in Dyestuff and Intermediates from Institute of Chemical Technology, India in 2011. His research focuses on using nanoparticles as drug delivery carriers for cancer treatment.

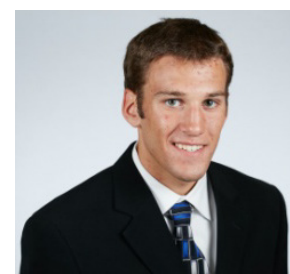

Derek VanDyke is currently a junior in the Department of Chemical Engineering at the University of Massachusetts Lowell. He has presented his research findings at the 250 th American Chemical

Society National Meeting for which he was awarded the American Chemical Society Travel Award, the 90th American Chemical Society Colloids Meeting, and will present at the 2016 American Institute of Chemical Engineers Annual Meeting.

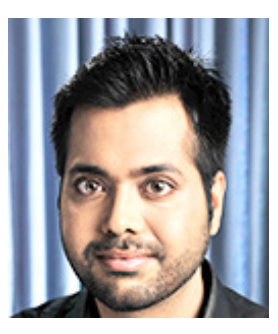

Dr. Prakash Rai is an assistant professor at the University of Massachusetts, Lowell in the department of chemical engineering. $\mathrm{He}$ received his BS in chemical engineering from the University of Mumbai, India in 2003. He obtained his MS and PhD degrees in chemical and biological engineering from Rensselaer Polytechnic Institute, Troy, NY in May and December of 2007 respectively. He is the author of more than 20 journal papers and one book chapter. His current research interests include nanotechnology, photodynamic therapy, fluorescence-based contrast agents, optical imaging, image-guided drug delivery and theranostics. He is a member of the American Institute of Chemical Engineers, American Physical Society, American Chemical Society, and the American Association of Cancer Research. 